EFICACIA Y SATISFACCIÓN DEL PROGRAMA RESILIENCIA Y BIENESTAR: QUÉDATE EN CASA. LA PSICOLOGÍA EN TIEMPOS DE CUARENTENA Y PANDEMIA

EFFICACY AND SATISFACTION OF THE RESILIENCE AND WELLBEING PROGRAM: «STAY AT HOME». PSYCHOLOGY IN TIMES OF QUARANTINE AND PANDEMIC

\title{
Óscar Sánchez-Hernández
}

Universidad de Murcia. España

ORCID: https://orcid.org/0000-0002-9501-1815

\section{Ana Canales}

Sander Psicólogos: Bienestar \& Ciencia. España ORCID: https://orcid.org/0000-0002-8659-3999

Cómo referenciar este artículo/How to reference this article:

Sánchez-Hernández, O. y Canales, A. (2020). Eficacia y Satisfacción del Programa Resiliencia y Bienestar: Quédate en casa. La Psicología en tiempos de Cuarentena y Pandemia. Revista de Psicoterapia, 31(117), 381-398. https://doi.org/10.33898/rdp.v31i117.389 


\title{
Resumen
}

El estado de alarma generado por la crisis del COVID-19 conllevó aplicar la cuarentena para la población española entre otros países. El Programa Resiliencia y Bienestar: "Quédate en casa”, es una intervención psicoeducativa que se aplicó en el confinamiento y desescalada con la finalidad de promocionar el bienestar y prevenir problemas emocionales. Se evaluó la eficacia y satisfacción del programa en una muestra de 80 participantes, 68,8\% mujeres y un 31,1\% hombres, con una media de edad de 36,04 años $(D E=15,1)$. Los participantes informaron de una alta satisfacción con el programa. Seguir comportamientos prudentes y saludables en el confinamiento (quedarse en casa, uso de mascarillas...) se relaciona con la satisfacción con el programa y con el desarrollo de las habilidades del programa. Los análisis señalan una mejora estadísticamente significativa, del pretest al postest, en resiliencia, bienestar psicológico, síntomas de estrés postraumático, facilidad para llevar la pandemia y estado de ánimo. Las mejoras se dieron también en participantes que no habían realizado otra intervención psicológica en el último mes (para descartar que la mejora fuera por otra intervención psicológica que hubieran recibido). El desarrollo de habilidades del programa predice la mejora, del pretest al postest, de la facilidad de llevar la pandemia y del bienestar psicológico. Se recomiendan nuevos análisis y ensayos en diseños experimentales para estudiar su eficacia.

Palabras clave: resiliencia, bienestar, pandemia, cuarentena, prevención, covid-19.

\begin{abstract}
The state of alarm generated by the COVID-19 crisis led to the application of quarantine for the Spanish population among other countries. The Resilience and Well-being Program: "Stay at home" is a psychoeducational intervention that was applied in confinement and de-escalation in order to promote well-being and prevent emotional problems. The efficacy and satisfaction of the program were evaluated in a sample of 80 participants, $68.8 \%$ women and $31.1 \%$ men, with a mean age of 36.04 years (SD 15.1). Participants reported high satisfaction with the program. Following prudent and healthy behaviors in confinement (staying home, wearing masks ...) is related to satisfaction with the program and the development of program skills. The analyzes indicate a statistically significant improvement, from pretest to posttest, in resilience, psychological well-being, symptoms of post-traumatic stress, ease of handling the pandemic, and mood. The improvements also occurred in participants who had not undergone another psychological intervention in the last month (to rule out that the improvement was not due to another psychological intervention they had received). The development of program skills predicts improvement, from pretest to posttest, in ease of handling the pandemic and psychological well-being. Further analyzes and trials in experimental designs are recommended to study its efficacy.
\end{abstract}

Keywords: resilience, well-being, pandemic, quarantine, prevention, covid-19. 
Eficacia y Satisfacción del Programa Resiliencia y Bienestar: «quédate en casa»

El estado de pandemia y cuarentena provocado por el COVID-19 ha supuesto un verdadero reto para todos los países, instituciones, organizaciones, familias y personas del planeta.

La revisión del grupo de investigación de Brooks y colaboradores (2020) en su artículo publicado "The psychological impact of quarantine and how to reduce it: rapid review of the evidence" explican el brote de la enfermedad por coronavirus de diciembre de 2019 y el efecto de la aplicación del confinamiento para la población general. Los autores del estudio señalan que las decisiones sobre cómo aplicar la cuarentena deben basarse en la mejor evidencia disponible. Hicieron una revisión del impacto psicológico de la cuarentena utilizando tres bases de datos. De 3166 artículos científicos encontrados, 24 fueron incluidos en la revisión. La mayoría de los estudios revisados informaron de efectos psicológicos negativos, incluidos síntomas de estrés postraumático, confusión y enojo. Los factores estresantes incluyeron una mayor duración de la cuarentena, temores de infección, frustración, aburrimiento, suministros inadecuados, información inadecuada, pérdidas financieras y estigma. Algunos investigadores han sugerido efectos duraderos.

Recomiendan los autores que en situaciones donde la cuarentena se considera necesaria, los estados deben poner en cuarentena a las personas por un tiempo no mayor al requerido, proporcionar una justificación clara para la cuarentena e información sobre los protocolos y garantizar que se proporcionen suministros suficientes. Subrayan especialmente apelar al altruismo recordando a la población los beneficios de la cuarentena para la sociedad en general lo que puede ser favorable para llevar mejor este proceso. Señalan los beneficios de proporcionar información adecuada para sobrellevar la cuarentena, estrategias de afrontamiento y gestión del estrés dado que el aburrimiento y el aislamiento pueden generar estrés y angustia. Subrayan estudios en los que se han encontrado beneficios de dar apoyo telefónico por expertos en salud mental específicamente para los que están en cuarentena en términos de proporcionarles una red social.

Diversos estudios señalan un impacto psicológico negativo de la cuarentena y pandemia del COVID-19 en China (Liang et al., 2020; Wang et al., 2020), España (Valiente et al., 2020) y otros países (Torales et al., 2020) en la línea del estudio de Brooks y colaboradores (2020).

La consecuencia de este impacto hace probable que muchos países experimenten una "ola" de trastornos mentales relacionados con COVID-19 como resultado de un aumento de los factores de riesgo vinculados a la pandemia como el aislamiento social, desempleo, preocupaciones económicas, estrés, violencia de pareja, estrés laboral, incertidumbre, dolor y pérdida. El enfoque "doble" utilizado para lidiar con el COVID-19 a nivel médico es un enfoque excelente para usar también para la salud mental. A nivel médico comprende, por un lado, tratamiento médico para los infectados incrementando la capacidad de respuesta sanitaria y, por otro, medidas de salud pública de prevención primaria de contagios (distancia de seguridad, uso 
de mascarillas, lavado de manos frecuente...). Trasladando este excelente plan para manejar los impactos en la salud mental supondría, por un lado, incrementar la capacidad sanitaria para ofrecer tratamiento psicológico a personas que presenten malestar significativo y/o un trastorno psicológico y, por otro lado, medidas de prevención primaria centradas en prevenir la aparición de nuevos casos de trastornos mentales. Un enfoque en la prevención primaria es particularmente importante para "aplanar la curva” y evitar un aumento en la incidencia de trastornos mentales derivados de la pandemia de COVID-19 (Carbone, 2020). Las intervenciones basadas en evidencia diseñadas para prevenir trastornos comunes ya están disponibles y deberían divulgarse y aplicarse (Arango et al., 2018; Extremera, 2020; Mendelson y Eaton, 2018; Sánchez-Hernández, 2012; Sánchez-Hernández et al., 2014). Estas intervenciones incluyen programas de crianza, sociales y emocionales, programas de aprendizaje, estrategias de autocuidado y programas de bienestar mental en el lugar de trabajo, entre otros. Gran parte de los programas de prevención de la depresión en niños y adolescentes han utilizado técnicas cognitivo-conductuales, con cierto éxito. Otras estrategias de prevención de la depresión han incluido entrenamiento en un adecuado afrontamiento de situaciones estresantes, habilidades de resolución de problemas sociales, entrenamiento en habilidades sociales, habilidades de comunicación, y fomento de adecuadas habilidades para la crianza de los hijos. Estas estrategias tienen en común que se dirigen hacia objetivos específicos, bien identificados, presentan la intervención en formato de manuales cuidando la fidelidad de dicha intervención y se centran principalmente en las dimensiones cognitiva y social. Las intervenciones de prevención de la depresión podrían ser una medida eficiente de ahorro sanitario (Mihalopoulos et al., 2011) por lo que es necesario invertir en este tipo de medidas.

En el pasado brote del síndrome respiratorio agudo severo (SARS) del 2003 el estudio de Maunder y colaboradores (2003) encontraron beneficios al proporcionar apoyo telefónico, por expertos en salud mental, a las personas en cuarentena en términos de proporcionarles una red social de apoyo. Identificar las necesidades de las familias, ofrecer una oportunidad para expresar sentimientos y apoyar estrategias de afrontamiento efectivas ayudó a mejorar el sentido de competencia y control de las familias.

Si nos centramos dentro de los problemas emocionales en la depresión, los estudios epidemiológicos y clínicos indican que los factores de riesgo más relacionados son: ser mujer, tener una historia familiar de depresión, especialmente uno o ambos padres, presentar síntomas depresivos (subclínicos), ansiedad, acontecimientos vitales estresantes, desarreglos neurobiológicos, temperamento difícil, rasgos de personalidad como neuroticismo, cogniciones negativas, problemas en la autorregulación y el afrontamiento y dificultades en las relaciones interpersonales (Garber, 2006).

Southwick et al. (2005) presentaron un valioso resumen de los factores protectores que contribuyen a la resistencia al estrés. Teniendo en cuenta el impacto 
del estrés en el inicio de la depresión, el aumento de estos factores de protección puede desempeñar una función primaria útil o coadyuvante en las intervenciones preventivas. Southwick y colaboradores (2005) señalaron los siguientes factores para la promoción de la resistencia al estrés según indica la investigación: la emocionalidad positiva, disposición al optimismo, el humor, la flexibilidad cognitiva, el estilo explicativo optimista, la capacidad de redefinir los factores de estrés, la aceptación, la espiritualidad, el altruismo, el apoyo social, presencia de modelos de conducta, el estilo de afrontamiento activo y el ejercicio físico.

Algunos de los factores de resiliencia más prácticos para ser modificados por una intervención incluyen el estilo de afrontamiento activo, la aceptación, y la capacidad de redefinir los factores de estrés (similar al estilo de atribución). Por ejemplo, la aceptación es probable que sea importante en las intervenciones en los que la persona no tiene el control de la situación. En cuanto al afrontamiento activo, algunos programas dirigidos a resolver problemas específicos han dado resultados prometedores en muestras de adultos.

Para la prevención de problemas emocionales en jóvenes el Programa de Resiliencia de Pensilvania es uno de los más evaluados con resultados muy positivos (Brunwasser et al., 2009; Sánchez-Hernández et al., 2016) y, en el ámbito español, el Programa Sonrisa: Psicología Basada en la Evidencia ha dado resultados alentadores (Sánchez-Hernández et al., 2019). Muchas de las técnicas de estos programas fueron utilizadas en el diseño del Programa Resiliencia y Bienestar: «Quédate en casa».

Las últimas revisiones meta-analíticas subrayan la necesidad intervenciones de la Psicología Positiva para promocionar el bienestar psicológico. En el estudio de Koydemir et al. (2020) se incluyeron 68 estudios controlados aleatorios de poblaciones no clínicas con un total de 16.085 participantes. Los resultados mostraron que las intervenciones psicológicas positivas aumentan el bienestar tanto a corto como a largo plazo.

\section{Programa Resiliencia y Bienestar: «Quédate en casa»}

Al igual que la medicina, creemos que la psicología debe responder a todos los niveles necesarios (promoción del bienestar, prevención de problemas emocionales y detección e intervención precoz). Desde la Universidad de Murcia y el Centro de Psicología Sander Psicólogos: Bienestar \& Ciencia, organizaciones a las que pertenecemos los autores, nos propusimos responder a este reto en base a nuestra experiencia profesional llevando a cabo el Programa Resiliencia y Bienestar: «Quédate en Casa» (Sánchez-Hernández y Canales, 2020) con dos objetivos claros:

1. Prevenir problemas emocionales que podría producir el estado de cuarentena y pandemia en la población, según las últimas revisiones científicas, y promocionar el bienestar de las familias.

2. Detección precoz y derivación a asociaciones y organizaciones que ofrezcan tratamiento psicológico gratuito en caso de que fuera necesario.

Es una intervención psicoeducativa que está pensada especialmente para 
afrontar de forma más sabia y saludable la situación de cuarentena, así como los efectos a corto, medio y largo plazo provocado tanto por la cuarentena como la pandemia que requerirá futuras adaptaciones que tengamos que realizar de las recomendaciones sanitarias.

Para su elaboración se ha tenido en cuenta las investigaciones previas sobre la prevención de problemas emocionales y la promoción del bienestar. Se tienen en cuenta tanto los factores de riesgo como los factores protectores con un enfoque ecológico (Brunwasser et al., 2009; Sánchez-Hernández et al., 2014; SánchezHernández et al., 2016; Sánchez-Hernández et al., 2019). Se siguieron las indicaciones de los expertos del Peking University Sixth Hospital (Bao et al., 2020) para que la población pueda lidiar con el estrés: evaluar la precisión de la información divulgada, mejorar los sistemas de apoyo social (familia, amigos...), eliminar el estigma asociado con la pandemia, mantener una vida normal en condiciones seguras y utilizar el sistema de servicios psicosociales (asesoramiento telefónico, videollamadas...).

También se han tenido en cuenta estudios e intervenciones para evitar los efectos negativos de procesos de confinamiento en otros contextos. Un ejemplo interesante son los entrenamientos de los astronautas con dinámicas de la psicología, en general, y de la psicología positiva en particular para fomentar sus emociones positivas ante las dificultades emocionales que supone el confinamiento. Los astronautas que preparan el viaje a Marte entrenan dinámicas muy parecidas a las de este programa. EARTH of Well-being se desarrolló en el marco del proyecto Mars-500 como una contramedida psicológica para ayudar a los astronautas a hacer frente a las dificultades durante el largo viaje simulado a Marte (520 días). Los datos apoyan la utilidad del sistema para aumentar las emociones positivas y disminuir las negativas (Botella et al., 2016).

El estudio que se presenta en este artículo forma parte del Proyecto Resiliencia y Bienestar que comprende tres fases. Las dos primeras se basan en la resiliencia reactiva y la tercera en la resiliencia proactiva (Jackson y Ferris, 2015). Tradicionalmente se ha hablado de una resiliencia reactiva, ya que se «reacciona» a la adversidad del entorno con una respuesta adaptativa positiva. La resiliencia proactiva consiste en adelantarse a los acontecimientos y proveer a las personas y organizaciones de los recursos psicológicos necesarios. Actuar focalizados en propósitos, objetivos y metas futuras (Salanova et al., 2019). Con esta explicación en mente las fases de este proyecto son:

1. Programa Resiliencia y Bienestar: «Quédate en Casa»

2. Programa Resiliencia y Bienestar: «Creando una Nueva Realidad»

3. Programa Resiliencia y Bienestar: «Construyendo una Comunidad Resiliente»

La resiliencia se refiere a la capacidad que la persona tiene de recuperarse después de sufrir una situación compleja, estresante, adaptándose de manera positiva y creativa a las adversidades, superándolas y, en general, sintiéndose regenerada, 
fortalecida y transformada para mejor. El término, al igual que el de estrés, proviene de la física y denota la capacidad de resistencia de un material al choque, a la tensión, a la presión y que le permite volver, siempre que es forzado o violentado, a su forma o posición inicial (Carrobles y Benevides-Pereira, 2009). De forma resumida es la capacidad de adaptación ante circunstancias cambiantes y adversas (Block y Block, 1980). La resiliencia es un factor protector que se relaciona con la promoción del bienestar y la prevención de problemas emocionales como señalan estudios realizados tras el confinamiento en la pandemia del COVID-19 (SánchezHernández et al., 2020). Si bien tendemos hacia la resiliencia (Bonanno, 2004), ante un evento masivo como el COVID-19, la prevención es un reto necesario a abordar. Aunque muchas personas y familias mostrarán resiliencia frente a los desafíos asociados con COVID-19, para muchos otros, el bloqueo prolongado y la falta de apoyo probablemente exacerbarán las vulnerabilidades existentes y contribuirán a la aparición de nuevos trastornos relacionados con el estrés (Horesh y Brown, 2020).

En resumen, este artículo evalúa la eficacia y satisfacción del Programa Resiliencia y Bienestar: «Quédate en casa» cuyos objetivos son la prevención de problemas emocionales, la promoción del bienestar y la detección e intervención precoz ante los efectos de la cuarentena y pandemia originada por el COVID-19. Por último, se proponen líneas de intervención e investigación para fortalecer comunidades mediante la resiliencia proactiva.

\section{Método}

\section{Participantes}

La muestra estuvo formada por 80 participantes, 68.8\% mujeres y un $31.2 \%$ hombres, con una media de edad de 36.04 años $(D E=15.1)$ principalmente con un nivel socioeconómico medio (88.8\%) y bajo en un $11.2 \%$. La mayoría de la muestra tiene estudios universitarios (66.3\%) y un $24.3 \%$ de la muestra informa, en el postest, de haber recibido apoyo psicológico en el último mes de forma simultánea al programa.

\section{Instrumentos}

Portada de Evaluación del Programa Resiliencia y Bienestar: «Quédate en casa». Recoge los datos sociodemográficos y los siguientes constructos medidos mediante un ítem en pretest y postest: facilidad para llevar la pandemia (“ ¿Hasta qué punto, como promedio de la última semana, te es fácil llevar esta situación de pandemia, de 0 a 10, siendo 0 muy difícil y 10 muy llevadero?”), y estado de ánimo (“¿Cómo puntuarías tu estado de ánimo promedio de la última semana, de 0 a 10, siendo 0 muy bajo estado de ánimo y 10 muy alto estado de ánimo?”). Constructos medidos sólo en postest: seguir los consejos del programa ("¿Hasta qué punto has seguido nuestros consejos, de 0 a 10 , siendo 0 muy poco y 10 mucho?”), satisfacción con el programa (“¿Qué nivel de satisfacción tienes con nuestra intervención, de 
0 a 10, siendo 0 muy poco y 10 mucho?”) y conocer si habían recibido otro apoyo psicológico (“¿Has recibido apoyo psicológico (aparte de este programa) durante el último mes?”).

Cuestionario Resumen de Habilidades del Programa Resiliencia y Bienestar: «Quédate en casa» (Sánchez-Hernández y Canales, 2020). Es un cuestionario de 15 ítems que pretende medir el nivel de aplicación, en una escala de 0 a 10 , de las distintas técnicas del programa: informarse adecuadamente de fuentes fiables, llevar hábitos y horarios saludables, uso de fortalezas, comportamientos saludables y prudentes, no exigirse de más a uno mismo ni a los demás, aceptar sabiamente las emociones, cultivar relaciones positivas y fomentar el apego seguro, agradecimiento y esperanza, técnica roca, optimismo resiliente, gestión de rumiaciones, mi mejor yo posible, sentido vital, dar sentido al sufrimiento y diario resiliente. La consistencia interna, alfa de Cronbach, fue de .84 .

Cuestionario de Resiliencia (Wagnild y Young, 1987). Adaptación de Baños y Botella (2017). Evalúa el grado de resiliencia individual y la perspectiva de resiliencia como una característica de personalidad positiva que favorece la adaptación (Wagnild y Young, 1993). Está formada por 25 ítems escritos de forma positiva, que se valoran del 1 (en desacuerdo) al 7 (totalmente de acuerdo), donde los resultados varían entre los 25 y 175 puntos. Según Wagnild y Young (1993), se consideran altas las puntuaciones en la escala cuando se obtienen puntuaciones iguales o superiores a 147 puntos. Además de poder calcular la puntuación total de la escala se pueden distinguir dos factores principales, que fueron nombrados "aceptación de la vida propia” y “competencia individual”. En una revisión de 12 artículos desarrollada por Wagnild (2009), la fiabilidad de la escala se encontraba entre .85 y .94. En nuestro estudio la consistencia interna, alfa de Cronbach, fue de .91.

Escala de Gravedad de Síntomas del Trastorno de Estrés Postraumático Revisada (EGS-R, Echeburúa, Amor, Sarasua, Zubizarreta, Holgado-Tello y Muñoz, 2016). Es una entrevista estructurada que consta de 21 ítems, basada en los criterios diagnósticos del DSM-5 para este trastorno: 5 hacen referencia a los síntomas de reexperimentación (rango de 0 a 15 puntos), 3 a los de evitación conductual/ cognitiva (rango de 0 a 9 puntos), 7 a alteraciones cognitivas y estado de ánimo negativo (rango de 0 a 21 puntos) y 6 a los síntomas de aumento de la activación y reactividad psicofisiológica (rango de 0 a 18 puntos). El rango de la escala global oscila de 0 a 63 puntos. La eficacia diagnóstica de la escala es muy alta (82.5\%) si se establece un punto de corte global de 20 . El instrumento global ha mostrado una alta consistencia interna $(\alpha=.91)$. En nuestro estudio la consistencia interna, alfa de Cronbach, fue de .93 .

Escala de Bienestar Mental de Warwick-Edimburgo (WEMWBS, Tennant et al., 2007). Adaptación española de López y colaboradores (2012). Esta medida aparece como consecuencia de una mayor demanda de instrumentos de medida que evalúen la salud mental positiva. Consta de 14 afirmaciones positivas referentes a aspectos hedónicos y eudaemónicos de bienestar mental. Cada ítem se responde 
sobre la base de una escala Likert de 5 puntos desde "nunca" hasta "todo el tiempo" y el resultado final se obtiene de la suma de todos los ítems (rango 14 a 70 puntos). Una mayor puntuación indica mayores niveles de bienestar mental. Ha sido validada al español en una muestra de 1900 participantes entre 15 y 70 años (Castellví et al., 2013), con alta consistencia interna (alfa de Cronbach $=.93$ ), buen poder discriminativo y ajuste satisfactorio para soluciones multifactoriales. En nuestro estudio la consistencia interna, alfa de Cronbach, fue de .83 .

\section{Procedimiento}

El estudio de la revisión realizada por Brooks y colaboradores (2020), sobre el impacto psicológico de las cuarentenas, fue el comienzo de este proyecto. El estado de cuarentena en España se inició el 14 de marzo. El 16 de marzo se ofreció el programa por redes sociales y a estudiantes de la Universidad de Murcia. En el primer contacto se informaba sobre el programa y las medidas de protección de datos y, a los interesados, se pedía el consentimiento informado.

La intervención se centró en el periodo de la cuarentena y desescalada. El 21 de junio el Gobierno de España concluye el último estado de alarma, terminada la desescalada y entrando todo el país en la 'nueva normalidad' tras 99 días de emergencia nacional. A los 80 participantes del programa se les realizó un pretest, al inicio de la intervención, y un postest tras la finalización de la misma.

Los profesionales que impartían la intervención tenían que ser psicólogos con especialidad en psicología sanitaria. Para formarse en la intervención tenían que pasar ellos mismos el programa como participantes antes de pasar la intervención a otras personas. También recibieron un curso de formación online de 4 horas por los creadores del programa, así como su supervisión durante sus intervenciones.

\section{Programa Resiliencia y Bienestar: «Quédate en casa»}

Es una intervención psicoeducativa que está pensada especialmente para afrontar de forma más sabia y saludable la situación de cuarentena, así como los efectos a corto, medio y largo plazo provocado tanto por la cuarentena como la pandemia que requerirá futuras adaptaciones que tengamos que realizar de las recomendaciones sanitarias.

El presente programa tiene un enfoque familiar. Todas las fichas pueden ser trabajadas por los adultos y algunas están más adaptadas a los más pequeños. En aquellas fichas que son para adultos, los padres o cuidadores principales pueden intentar extraer el mejor aprendizaje y transmitir su mensaje adaptado al lenguaje de los más pequeños. Consideramos que lo fundamental es que los adultos, padres y cuidadores puedan gestionar lo más sabiamente esta situación y sepan cuidarse a sí mismos para poder cuidar de las personas a su cargo fomentando el apego seguro (Hoffman et al., 2019; Powell et al., 2019; Yap et al., 2016).

El programa ofrece apoyo psicológico gratuito con tres sesiones de videollamada por persona (20-30 minutos cada sesión) y envío de 7 píldoras de bienestar 
mediante correo electrónico en la línea de otras intervenciones (Hansen et al., 2019).

Pueden escucharse las recomendaciones de cada píldora de bienestar en Radio UM.es, la radio de la Universidad de Murcia (https://www.ivoox.com/podcastprograma-resiliencia-bienestar-emocional-queda_sq_f1884376_1.html).

La intervención psicoeducativa propuesta integra diversos enfoques de la psicología:

1. Psicoeducación para informarse de fuentes fiables, fomento de la motivación altruista para comprometerse con las recomendaciones sanitarias, cuentoterapia para los más pequeños e información sobre la resiliencia (Píldora de Bienestar 1).

2. Enfoque Cognitivo-Conductual y Activación Conductual con las técnicas de planificación de actividades agradables y saludables (Píldora de Bienestar 2), respiración profunda, imágenes positivas (Píldora de Bienestar 3) estilo de comunicación asertivo y habilidades de resolución de problemas (Píldora de Bienestar 4).

3. Educación Emocional con técnicas centradas en percepción, expresión, comprensión y regulación emocional (Píldora de Bienestar 3 y 4).

4. Terapia Cognitiva con técnicas de reestructuración cognitiva y afrontamiento de las rumiaciones (Píldora de Bienestar 6).

5. Terapia de Aceptación y Compromiso, Mindfulness y Terapia Centrada en la Compasión con técnicas de atención plena, defusión cognitiva, compromiso con los valores y técnicas centradas en la compasión (Píldoras de Bienestar 3, 4, 5, 6 y 7).

6. Técnicas corporales centrados en los estudios sobre posturas poderosas de Amy Cuddy (2016), profesora en la Harvard Business School, así como en otras autoras (Ogden y Fisher, 2016) en la Píldoras de Bienestar 3.

7. Terapia de Apego centrada en los consejos de Powell et al. (2019) sobre el Círculo de Seguridad (Píldoras de Bienestar 4).

8. Psicología Positiva Aplicada con las técnicas de identificación y desarrollo de fortalezas psicológicas, esperanza, saboreo, optimismo resiliente y sentido vital (Píldoras de Bienestar 2, 4, 5, 6 y 7).

El programa también tiene como objetivo la detección precoz de problemas emocionales y la derivación a asociaciones y organizaciones que realicen intervención psicológica gratuita en situación de crisis y emergencias. Tanto en el pretest como en el postest se usó como criterio de derivación el punto clínico de 20 de la Escala de Gravedad de Síntomas del Trastorno de Estrés Postraumático Revisada (EGS-R, Echeburúa et al., 2016) para informar a los participantes de la idoneidad de ponerse en contacto con estas organizaciones en la línea de otras intervenciones (Zhou et al., 2020).

La primera recomendación fue la propuesta de la Asociación EMDR España. La terapia EMDR (Desensibilización y Reprocesamiento por los Movimientos Oculares) está avalada por la Organización Mundial de la Salud y las Guías Clínicas 
Internacionales para el tratamiento del trauma (Hofman, 2016).

Se basa en la comprensión del efecto de las experiencias vitales adversas y traumáticas sobre la patología y en el procesamiento de dichas experiencias a través de procedimientos estructurados que incluyen movimientos oculares u otras formas de estimulación bilateral. Su aplicación se ha extendido a un amplio rango de problemas clínicos (https://www.emdr-es.org/).

Los análisis estadísticos realizados se centraron en análisis de correlaciones, análisis de regresión lineal, cálculo de las puntuaciones $t$ de Student para medias relacionadas y cálculo del tamaño del efecto (Cohen, 1988; Hedges, 1981).

\section{Resultados}

\section{Satisfacción con el programa}

Los participantes informaron de una alta satisfacción con el programa con una media de 8.26 ( $D E=1.3$ ) y que habían seguido los consejos del programa de forma notable señalando una media de $7.25(D E=1.7)$. La satisfacción con el programa se relacionaba con el desarrollo de las habilidades del programa $(r=.45 ; p=.002)$ y haber seguido sus consejos $(r=.43 ; p=.000)$.

\section{Comportamientos prudentes y saludables}

Seguir comportamientos prudentes y saludables (quedarse en casa, uso de mascarillas, distancia social, lavado de manos y seguir recomendaciones sanitarias en general) se relaciona con la satisfacción con el programa $(r=.39 ; p=.008)$ y con el desarrollo de las habilidades del programa, específicamente con, seguir fuentes fiables de información y evitar el exceso de información $(r=.52 ; p=.000)$, uso de fortalezas $(r=.34 ; p=.022)$, relaciones positivas y apego seguro $(r=.35$; $p=.015)$, esperanza y agradecimiento $(r=.29 ; p=.045)$, optimismo resiliente $(r$ $=.31 ; p=.036)$, sentido vital $(r=.30 ; p=.044)$ y llevar un diario resiliente $(r=$ $.42 ; p=.003)$.

\section{Análisis de mejoras del pretest al postest muestra total}

Los análisis señalan una mejora estadísticamente significativa, del pretest al postest, en la facilidad para llevar la pandemia, el estado de ánimo, los síntomas de estrés postraumático, el bienestar psicológico y en la resiliencia (tabla 1) (figura 1). 
Tabla 1. Descriptivos (Medias y Desviaciones Estandar) y Puntuaciones T de Student, del Pretest al Postest, y Tamaños del Efecto en la Muestra Total

\begin{tabular}{|c|c|c|c|c|c|c|}
\hline \multirow[t]{2}{*}{ Variable } & \multirow{2}{*}{$\begin{array}{c}\text { Programa } \\
\text { Media }\end{array}$} & \multicolumn{2}{|c|}{ Resiliencia } & \multirow[t]{2}{*}{$\mathrm{t}(\mathrm{gl})$} & \multirow[t]{2}{*}{$\mathrm{p}$} & \multirow[t]{2}{*}{$\begin{array}{l}\text { Tamaño } \\
\text { del efecto }\end{array}$} \\
\hline & & $\mathrm{n}$ & DE & & & \\
\hline \multicolumn{7}{|c|}{$\begin{array}{c}\text { Facilidad de llevar la } \\
\text { pandemia }\end{array}$} \\
\hline Pretest & 6.45 & 74 & 1.84 & -- & & \\
\hline Postest & 7.73 & 74 & 1.61 & $-5.12(73)$ & .000 & .70 \\
\hline \multicolumn{7}{|c|}{ Estado de Ánimo } \\
\hline Pretest & 6.05 & 73 & 1.76 & -- & & \\
\hline Postest & 7.27 & 73 & 1.49 & $-5.27(72)$ & .000 & .70 \\
\hline \multicolumn{7}{|c|}{ Estrés Postraumático } \\
\hline Pretest & 18.70 & 53 & 11.99 & -- & & \\
\hline Postest & 13.66 & 53 & 11.06 & $3.93(52)$ & .000 & .41 \\
\hline \multicolumn{7}{|c|}{ Bienestar } \\
\hline Pretest & 47.59 & 51 & 7.37 & -- & & \\
\hline Postest & 51.92 & 51 & 8.73 & $-3.51(50)$ & .001 & .58 \\
\hline \multicolumn{7}{|c|}{ Resiliencia } \\
\hline Pretest & 134.74 & 50 & 19.20 & -- & & \\
\hline Postest & 138.96 & 50 & 16.75 & $-2.16(49)$ & .035 & .22 \\
\hline
\end{tabular}


Figura 1. Mejoras del Pretest al Postest en la Muestra Total

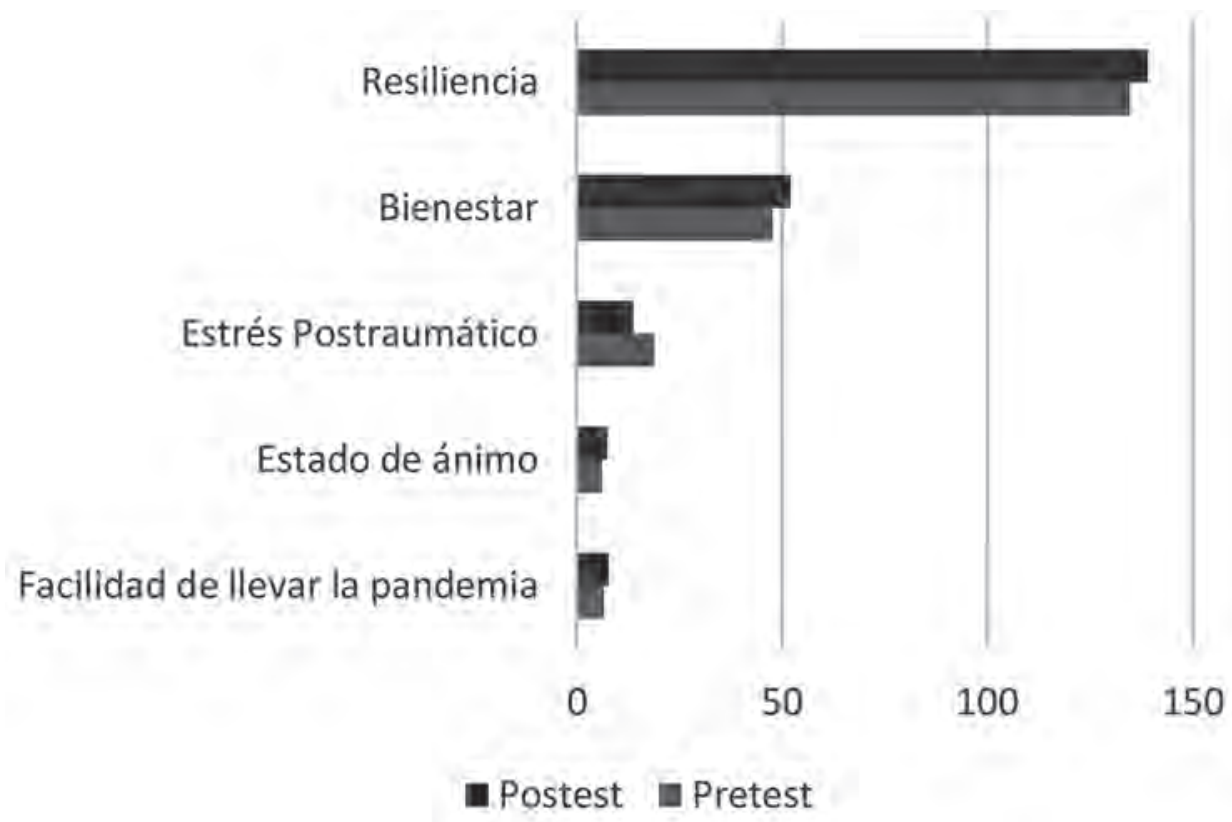

Análisis de mejoras del pretest al postest en los participantes que solo recibieron el programa

Las mejoras se dieron también en participantes que no habían realizado otra intervención psicológica en el último mes. Estos análisis tenían la finalidad de descartar que la mejora fuera por otra intervención psicológica que hubieran recibido. Los análisis señalan una mejora estadísticamente significativa, del pretest al postest, en la facilidad para llevar la pandemia, el estado de ánimo, los síntomas de estrés postraumático, el bienestar psicológico y marginalmente significativo en resiliencia (tabla 2). 
Tabla 2. Descriptivos (Medias y Desviaciones Estandar) y Puntuaciones T de Student, del Pretest al Postest, y Tamaños del Efecto en Particpantes que solo Recibieron el Programa

\begin{tabular}{|c|c|c|c|c|c|c|}
\hline \multirow[t]{2}{*}{ Variable } & \multirow{2}{*}{$\begin{array}{c}\text { Programa } \\
\text { Media }\end{array}$} & \multicolumn{2}{|c|}{ Resiliencia } & \multirow[t]{2}{*}{$t(\mathrm{gl})$} & \multirow[t]{2}{*}{$p$} & \multirow[t]{2}{*}{$\begin{array}{l}\text { Tamaño } \\
\text { del efecto }\end{array}$} \\
\hline & & $\mathrm{n}$ & DE & & & \\
\hline \multicolumn{7}{|c|}{$\begin{array}{c}\text { Facilidad de Ilevar la } \\
\text { pandemia }\end{array}$} \\
\hline Pretest & 6.59 & 54 & 2.02 & -- & & \\
\hline Postest & 8.00 & 54 & 1.33 & $-5.04(53)$ & .000 & .70 \\
\hline \multicolumn{7}{|c|}{ Estado de Ánimo } \\
\hline Pretest & 6.34 & 53 & 1.87 & -- & & \\
\hline Postest & 7.42 & 53 & 1.57 & $-3.74(52)$ & .000 & .57 \\
\hline \multicolumn{7}{|c|}{$\begin{array}{c}\text { Estrés } \\
\text { Postraumático }\end{array}$} \\
\hline Pretest & 16.34 & 38 & 10.79 & -- & & \\
\hline Postest & 11.08 & 38 & 8.55 & $3.97(37)$ & .000 & .48 \\
\hline \multicolumn{7}{|l|}{ Bienestar } \\
\hline Pretest & 47.78 & 37 & 7.24 & -- & & \\
\hline Postest & 52.59 & 37 & 8.17 & $-3.24(36)$ & .003 & .65 \\
\hline \multicolumn{7}{|c|}{ Resiliencia } \\
\hline Pretest & 137.92 & 36 & 20.59 & -- & & \\
\hline Postest & 142.14 & 36 & 17.06 & $-1.83(35)$ & .075 & .20 \\
\hline
\end{tabular}

Análisis de regresión sobre las puntuaciones de cambio

La finalidad de estos análisis era determinar hasta qué punto la mejora (puntuaciones de cambio del pretest al postest) en las diversas variables (facilidad de llevar la pandemia, estado de ánimo, estrés postraumático, bienestar y resiliencia) era producido por el desarrollo de las habilidades del programa. Los análisis de regresión señalan que el desarrollo de habilidades del programa predice la mejora, del pretest al postest, en la facilidad de llevar la pandemia (32\%) y del bienestar psicológico (49\%) de forma estadísticamente significativa (tabla 3). 
Tabla 3. Análisis de Regresión Utilizando las Distintas Dimensiones del Desarrollo de Habilidades del Programa como Variables Predictoras de las Puntuaciones de Cambio de las Diversas Variables (Facilidad de Llevar la Pandemia, Estado de Ánimo, Estrés Postraumático, Bienestar y Resiliencia)

\begin{tabular}{ccccccc}
\hline $\begin{array}{c}\text { Desarrollo de } \\
\text { Habilidades del } \\
\text { Programa }\end{array}$ & $\mathbf{R}$ & $\mathbf{R}^{2}$ & $\mathbf{R}^{2}$ corregida & $\begin{array}{c}\text { Error típico de } \\
\text { Estimación }\end{array}$ & $\mathbf{F}$ & $\mathbf{p}$ \\
\hline $\begin{array}{c}\text { Facilidad de llevar la } \\
\text { pandemia }\end{array}$ & .753 & .566 & .316 & 1.770 & 2.265 & .033 \\
\hline Estado de Ánimo & .664 & .441 & .119 & 1.879 & 1.369 & .234 \\
\hline Estrés Postraumático & .754 & .568 & .208 & 8.502 & 1.579 & .177 \\
\hline Bienestar & .873 & .761 & .486 & 7.049 & 2.765 & .036 \\
\hline Resiliencia & .722 & .521 & .072 & 12.584 & 1.159 & .385 \\
\hline
\end{tabular}

\section{Discusión y conclusiones}

Los participantes que pasaron el Programa Resiliencia y Bienestar: "Quédate en casa” informaron de una alta satisfacción con el programa. La satisfacción con el programa se relacionaba con el desarrollo de las habilidades del programa y haber seguido sus consejos.

Seguir comportamientos prudentes y saludables (quedarse en casa, uso de mascarillas, distancia social, lavado de manos y seguir recomendaciones sanitarias en general) se relaciona con la satisfacción con el programa y con el desarrollo de las habilidades del programa (seguir fuentes fiables de información, uso de fortalezas, relaciones positivas y apego seguro, esperanza, agradecimiento, optimismo resiliente, sentido vital y llevar un diario resiliente).

Los análisis señalan una mejora estadísticamente significativa, del pretest al postest, en resiliencia, bienestar psicológico, síntomas de estrés postraumático, facilidad para llevar la pandemia y estado de ánimo. Las mejoras se dieron también en participantes que no habían realizado otra intervención psicológica en el último mes (para descartar que la mejora fuera por otra intervención psicológica que hubieran recibido). El desarrollo de habilidades del programa predice la mejora, del pretest al postest, en la facilidad de llevar la pandemia y del bienestar psicológico.

Entre las limitaciones del estudio cabe señalar que el diseño fue preexperimental y tan sólo un diseño experimental puede garantizar que la causa de la mejora en los participantes es debido al programa. Dado que los análisis realizados señalan que el programa podría tener beneficios en promocionar el bienestar y prevenir problemas emocionales, se sugiere nuevos estudios con un diseño experimental. No obstante, los análisis que descartan que la mejora sea por otras intervenciones psicológicas y 
los que indican que el desarrollo de habilidades del programa predice las mejoras en la facilidad de llevar la pandemia y del bienestar psicológico apuntan hacia la eficacia del programa.

Se realizó una revisión bibliográfica buscando intervenciones psicológicas para prevenir problemas emocionales y promocionar el bienestar ante el efecto de la cuarentena en la crisis del COVID-19 pero tan sólo se hallaron artículos recomendando la necesidad de realizar estas intervenciones (Carbone, 2020; Fontanesi et al., 2020).

En el estudio de Maunder y colaboradores (2003) ante el brote del síndrome respiratorio agudo severo (SARS) del 2003 encontraron beneficios al proporcionar apoyo telefónico, por expertos en salud mental, a las personas en cuarentena en términos de proporcionarles una red social de apoyo. Identificar las necesidades de las familias, ofrecer una oportunidad para expresar sentimientos y apoyar estrategias de afrontamiento efectivas ayudó a mejorar el sentido de competencia y control de las familias.

Se recomienda la promoción de la Educación en Resiliencia, tanto reactiva como proactiva, dados los efectos positivos en la promoción del bienestar y la prevención de problemas emocionales. Estas medidas deben de ir acompañadas de la detección precoz y tratamiento psicológico de las personas que lo necesiten, atendiendo especialmente a los grupos más vulnerables. De igual forma medidas sociales que ayuden a amortiguar los efectos de esta crisis.

\section{Referencias}

Arango, C., Díaz-Caneja, C. M., McGorry, P. D., Rapoport, J., Sommer, I. E., Vorstman, J. A., McDaid, D., Marín, O., Serrano-Drozdowskyj, E., Freedman, R. y Carpenter, W. (2018). Preventive strategies for mental health. The Lancet. Psychiatry, 5(7), 591-604. https://doi.org/10.1016/S2215-0366(18)30057-9

Bao, Y., Sun, Y., Meng. S, Shi, J,yLu, L. (2020). 2019-nCoV epidemic: address mental health care to empower society. Lancet, 395(10224): e37-e38. https://doi.org/10.1016/s0140-6736(20)30309-3

Block, J. H. y Block, J. (1980). The role of ego-control and ego-resiliency in the organization of behaviour. En W. A. Collins (ed.), Minesota Symposia on Child Psychology: Volumen 13. Development of cognition, affect, and social relations (pp. 39-101). Erlbaum.

Bonanno, G. A. (2004). Loss, trauma, and human resilience: Have we underestimated the human capacity to thrive after extremely aversive events? American Psychologist, 59(1), 20-28. https://doi.org/10.1037/0003066X.59.1.20

Botella, C., Baños, R. M., Etchemendy, E., García-Palacios, A. y Alcañiz, M. (2016). Psychological countermeasures in manned space missions: "EARTH" system for the mars-500 project. Computers in Human Behavior, 55(B), 898-908. https://doi.org/10.1016/j.chb.2015.10.010

Brooks, S. K., Webster, R. K., Smith, L. E., Woodland, L., Wessely, S., Greenberg, N. y James, G. (2020). The psychological impact of quarantine and how to reduce it: Rapid review of the evidence. Lancet, 395(10227), 912-920. https://doi.org/10.1016/S0140-6736(20)30460-8

Brunwasser, S. M., Gillham, J. E. y Kim, E. S. (2009). A meta-analytic re-view of the Penn Resiliency Program's effect on depressive symptoms. Journal of Consulting and Clinical Psychology, 77(6), 1042-1054. https:// doi.org/10.1037/a0017671

Carbone, S. R. (2020). Flattening the curve of mental ill-health: the importance of primary prevention in managing the mental health impacts of COVID-19. Mental Health \& Prevention, 19, 200185. https://doi. org/10.1016/j.mhp.2020.200185 
Carrobles, J. A. y Benevides-Pereira, A. M. T. (2009): El estrés y la psicología positiva. En E. G. FernándezAbascal, (ed.), Emociones positivas (pp. 363-373). Pirámide.

Castellví, P., Forero, C. G., Codony, M., Vilagut, G., Brugulat, P., Medina, A., Gabilondo, A., Mompart, A., Colom, J., Tresserras, R., Ferrer, M., Stewart-Brown, S. y Alonso, J. (2013). The Spanish Version of the Warwick-Edinburgh Mental Well-Being Scale (WEMWBS) is Valid for Use in the General Population. Quality of Life Research, 22(6), 1-12. https://doi.org/10.1007/s11136-013-0513-7

Cohen, J. (1988). Statistical power analysis for the behavioral sciences (2. $\left.{ }^{\mathrm{a}} \mathrm{ed}\right)$. Erlbaum.

Cuddy, A. (2016). El poder de la Presencia. Urano.

Echeburúa, E., Amor, P. J., Sarasua, B., Zubizarreta, I., Holgado-Tello, F. P., Muñoz, J.M. (2016). Escala de Gravedad de Síntomas Revisada (EGS-R) del Trastorno de Estrés Postraumático según el DSM-5: propiedades psicométricas. Terapia psicológica, 34(2), 111-128. http://dx.doi.org/10.4067/S0718-48082016000200004

Extremera, N. (2020). Afrontando el estrés causado por la pandemia del COVID-19: Futura agenda de investigación desde la inteligencia emocional. International Journal of Social Psychology, 35(3), 631-638. https://doi. org/10.1080/02134748.2020.1783857

Fontanesi, L., Marchetti, D., Mazza, C., Di Giandomenico, S., Roma, P. y Verrocchio, M. C. (2020). The effect of the COVID-19 lockdown on parents: A call to adopt urgent measures. Psychological Trauma: Theory, Research, Practice, and Policy, 12(S1), S79-S81. http://dx.doi.org/10.1037/tra0000672

Garber, J. (2006). Depression in Children and Adolescents. Linking Risk Research and Prevention. American Journal of Preventive Medicine, 31(6S1), 104-125. https://doi.org/10.1016/j.amepre.2006.07.007

Hansen, A., Broomfield, G. y Yap, M. B. H. (2019). A systematic review of technology assisted parenting programs for mental health problems in youth aged 0-18 years: Applicability to underserved Australian communities. Australian Journal of Psychology, 71(4), 433-462. https://doi.org/10.1111/ajpy.12250

Hedges, L. V. (1981). Distribution Theory for Glass's Estimator of Effect Size and Related Estimators. Journal of Educational Statistics, 6(2), 107-112. https://doi.org/10.3102\%2F10769986006002107

Hofman, A. (2016). EMDR. La terapia para los síndromes de estrés psicológico derivados del trauma. Guía práctica para el tratamiento de las personas traumatizadas. Ediciones EMDR Biblioteca.

Hoffman, K., Cooper, G., Powell, B. y Benton, C. M. (2019). Cómo criar un niño seguro. Cómo el Círculo de Seguridad de los Padres puede ayudar a la formación de vínculos afectivos, a su resiliencia emocional y a la libertad de exploración. Medici.

Horesh, D. y Brown, A. D. (2020). Traumatic stress in the age of COVID-19: A call to close critical gaps and adapt to new realities. Psychological Trauma: Theory, Research, Practice, and Policy, 12(4), 331-335. http://dx.doi.org/10.1037/tra0000592

Jackson, S. y Ferris, T. (2015). Proactive and Reactive Resilience: A Comparison of Perspectives. INCOSE Insight, 18,7 .

Koydemir, S., Sökmez, A. B. y Schütz, A. (2020). A Meta-Analysis of the Effectiveness of Randomized Controlled Positive Psychological Interventions on Subjective and Psychological Well-Being. Applied Research in Quality of Life. https://doi.org/10.1007/s11482-019-09788-Z

Liang, L., Ren, H., Cao, R., Hu, Y., Qin, Z., Li, C. y Mei, S. (2020). The Effect of COVID-19 on Youth Mental Health. Psychiatric Quarterly, 91, 841-852. https://doi.org/10.1007/s11126-020-09744-3

López, M. A., Gabilondo, A., Codony, M., García-Forero, C., Vilagut, G., Castellví, P., Ferrer, M. y Alonso, J. (2013). Adaptation into Spanish of the Warwick-Edinburgh Mental Well-being Scale (WEMWBS) and Preliminary Validation in a Student Sample. Quality of Life Research, 22, 1099-1104. https://doi. org/10.1007/s11136-012-0238-z

Maunder, R., Hunter, J., Vincent, L., Bennett, J., Peladeau, N., Leszcz, M., Sadavoy, J., Verhaeghe, L. M., Steinberg, R. y Mazzulli, T. (2003). The immediate psychological and occupational impact of the 2003 SARS outbreak in a teaching hospital. CMAJ, 168(10), 1245-1251.

Mendelson, T. y Eaton, W. W. (2018). Recent advances in the prevention of mental disorders. Social psychiatry and psychiatric epidemiology, 53(4), 325-339. https://doi.org/10.1007/s00127-018-1501-6

Mihalopoulos, C., Vos, T., Pirkis, J., Smit, F. y Carter, R. (2011). Do indicated preventive interventions for depression represent good value for money? Australian and New Zealand Journal of Psychiatry, 45(1), 36-44. https://doi.org/10.3109/00048674.2010.501024

Ogden, P. y Fisher, J. (2016). Psicoterapia Sensoriomotriz. Intervenciones para el trauma y el apego. Declée de Brouwer.

Powell, B., Cooper, G., Hoffman, K. y Marvin, B. (2019). La intervención del Círculo de Seguridad. Cómo mejorar el apego en las relaciones entre padres e hijos mediante la intervención temprana. Eleftheria.

Salanova, M., Llorens, S. y Martínez, I. M. (2019). Organizaciones Saludables. Una mirada desde la Psicología Positiva. Editorial Aranzadi. 
Sánchez-Hernández, Ó. (2012). Eficacia de sendas intervenciones de Psicología Positiva para la promoción del bienestar y la prevención de la depresión infantil y de la depresión adolescente [Tesis doctoral, Universidad de Murcia (España)]. Digitum. http://hdl.handle.net/10201/33094

Sánchez-Hernández, Ó. y Canales, A. (2020). ¡Quédate en casa! Resiliencia y Bienestar. Psicología Aplicada a tiempos de Pandemia. Editorial UOC.

Sánchez-Hernández, Ó., Méndez, F. X., Ato, M. y Garber, J. (2019). Prevention of Depressive Symptoms and Promotion of Well-being in Adolescents: A Randomized Controlled Trial of the Smile Program. Anales de Psicología, 35(2), 225-232. https://doi.org/10.6018/analesps.35.2.342591

Sánchez-Hernández, O., García, M. F. y Canales, A. (2020). Resiliencia y Bienestar en la prevención de problemas emocionales ante el COVID-19 [Comunicación]. 8th International Congress of Educational Sciences and Development, Pontevedra, España.

Sánchez-Hernández, Ó., Méndez, F. X. y Garber, J. (2014). Prevención de la depresión en niños y adolescentes: Revisión y reflexión. Revista de Psicopatología y Psicología Clínica, 19(1), 63-76. https://doi.org/10.5944/ rppc.vol.19.num.1.2014.12983

Sánchez-Hernández, Ó., Méndez, F. X. y Garber, J. (2016). Promoting resilience in children with depressive symptoms. Anales de Psicología, 32(3), 741-748

Southwick, S. M., Vythilingam, M. y Charney, D. S. (2005). The psychobiology of depression and resilience to stress: Implications for prevention and treatment. Annual Review of Clinical Psychology, 1, 255-291. https://doi.org/10.1146/annurev.clinpsy.1.102803.143948

Tennant, R., Hiller, L., Fishwick, R., Platt, S., Joseph, S., Weich, S., Parkinson, J., Secker, J. y Stewart-Brown, S. (2007). The Warwick-Edinburgh Mental Well-being Scale (WEMWBS): development and UK validation. Health and Quality of Life Outcomes, 5(63), 1477-7525. https://doi.org/10.1186/1477-7525-5-63

Torales, J., O’Higgins, M., Castaldelli-Maia, J. M. y Ventriglio, A. (2020). The outbreak of COVID-19 coronavirus and its impact on global mental health. International Journal of Social Psychiatry, 66(4), 317-320. https:// doi.org/10.1177/0020764020915212 20764020915212

Valiente, C., Vázquez, C., Peinado, V., Contreras, A., Trucharte, A., Bentall, R. y Martínez, A. (2020). VIDACOVID-19. Estudio nacional representativo de las respuestas de los ciudadanos de España ante la crisis de Covid-19: respuestas psicológicas. Resultados preliminares. Síntomas de ansiedad, depresión y estrés postraumático ante el COVID-19: prevalencia y predictores (Informe técnico 2.0.02/05/2020). Universidad Complutense de Madrid y Universidad de Sheffield. https:/www.ucm.es/inventap/file/vida-covid19-informe-ejecutivomalestar3520-final-1

Wagnild, G. (2009). A review of the Resilience Scale. Journal of Nursing Measurement, 17(2), 105-113.

Wagnild, G. y Young, H. (1987). The Resilience scale. Manuscrito no publicado.

Wagnild, G. y Young, H. (1993). Development and psychometric evaluation of the Resilience Scale. Journal of Nursing Measurement, 1(2), 165-178.

Wang, C., Pan, R., Wan, X., Tan, Y., Xu, L., Ho, C. S. y Ho, R. C. (2020). Immediate psychological responses and associated factors during the initial stage of the 2019 Coronavirus Disease (COVID-19) Epidemic among the general population in China. International Journal of Environmental Research and Public Health, 17(5), 1729. https://doi.org/10.3390/ijerph17051729

Yap, M. B. H., Morgan, A. J., Cairns, K., Jorm, A. F., Hetrick, S. E. y Merry, S. (2016). Parents in prevention: A meta-analysis of randomized controlled trials of parenting interventions to prevent internalizing problems in children from birth to age 18. Clinical Psychology Review, 50, 138-158. https://doi.org/10.1016/j. cpr.2016.10.003

Zhou, X., Snoswell, C. L., Harding, L. E., Bambling, M., Edirippulige, S., Bai, X. y Smith, A. C. (2020). The Role of Telehealth in Reducing the Mental Health Burden from COVID-19. Telemedicine and e-Health, 26(4), 377-379. https://doi.org/10.1089/tmj.2020.0068 